\title{
EDITORIAL: The Many Challenges to the Field of Pediatric Pulmonology Posed by Pediatric Pulmonary Hypertension and the Path Forward
}

\author{
George Mallory $^{1}$ and Steven Abman ${ }^{2}$ \\ ${ }^{1}$ Baylor College of Medicine \\ ${ }^{2}$ University of Colorado Denver School of Medicine
}

December 12, 2020

\begin{abstract}
Pulmonary hypertension represents an increasingly important group of pediatric patients which commonly come to the attention, if not the primary care of pediatric pulmonologists around the world. There have been major advances in diagnosis and therapy over the past 25 years. To address potential gaps in knowledge, the authors were invited by the Editor of Pediatric Pulmonology to organize a series of manuscripts in a special supplement of the journal. Our authors include pulmonologists, pharmacists, intensivists, mid-level practitioners, neonatologists and cardiologists. We believe that this issue will be of great interest to most of the readership community that the Journal addresses.
\end{abstract}

EDITORIAL: The Many Challenges to the Field of Pediatric Pulmonology Posed by Pediatric Pulmonary Hypertension and the Path Forward

Authors: George B Mallory Jr., MD, Division of Pediatric Pulmonology, Baylor College of Medicine, Texas Children's Hospital, Houston, TX

Steven H. Abman, MD, Pediatric Heart Lung Center, Division of Pulmonary Medicine, Department of Pediatrics, University of Colorado School of Medicine, Children's Hospital Colorado, Aurora, COCorresponding author :

George B. Mallory Jr., MD

Mailing address: Section of Pediatric Pulmonology

Texas Children's Hospital

6701 Fannin Street, Suite 1040

Houston, TX 77030 Email: gbmallor@texaschildrens.org

Key words: pulmonary hypertension, pulmonary arterial hypertension, epidemiology, off-label drug use

Introduction:

Pediatric pulmonary hypertension ( $\mathrm{PH}$ ) has emerged as a major clinical problem throughout the world. This problem has grown for two reasons: a) increased awareness of the strong impact of $\mathrm{PH}$ on short and long-term outcomes in more diverse diseases, which has lead to an increase in screening and diagnostic evaluations; and b) the availability of PH-specific drug therapies leading to improved survival. ${ }^{1,2}$ We have the honor of leading off this special issue of Pediatric Pulmonology with an overview of the many faces of $\mathrm{PH}$ in neonates, infants, children and adolescents, with particular emphasis on the importance and need for 
pediatric pulmonologists to develop a greater understanding of $\mathrm{PH}$. While a large proportion of leaders in adult $\mathrm{PH}$ are pulmonologists, pediatric cardiologists have dominated the field in children with $\mathrm{PH}$. With growing awareness of the importance of $\mathrm{PH}$ in dictating outcomes of many childhood lung diseases and the critical contribution of respiratory problems to outcomes of children with many forms of $\mathrm{PH}$, it is clear that pediatric pulmonary specialists must become more knowledgeable and engaged in PH-related diseases. As reflected by the development of $\mathrm{PH}$ teams at many medical centers, it is evident that optimal care for these patients requires the collaboration of pediatric pulmonologists with pediatric cardiologists intensivists, neonatologists, pharmacists and others. ${ }^{2-4}$ As a result, the goal of this supplemental issue of Pediatric Pulmonology is to highlight and provide updates on key aspects of this important clinical field of pediatric PH.DEFINITIONS:

Pulmonary arterial hypertension (PAH) is defined as a patient with mean pulmonary artery pressure (PAP) $>20 \mathrm{mmHg}$, pulmonary capillary wedge pressure (PWCP) [?] $15 \mathrm{mmHg}$ and pulmonary vascular resistance index (PVRI) [?] $3 \mathrm{WU} \mathrm{m}^{2}$ in individuals older than three months of age. ${ }^{1}$ Pulmonary hypertension (PH) with or without variable degrees of pulmonary vascular disease (PVD) can also occur in patients with conditions associated with post-capillary diseases of elevated mean PAP with elevated pulmonary capillary wedge or left atrial pressures, as in the setting of left heart disease (Nice Group 2; Table 1) or high pulmonary blood flow in the setting of congenital heart disease with marked left-to-right shunts as with atrial and ventricular septal defects or with a large patent ductus arteriosus. In addition, significant PVD may be present and complicate outcomes in the absence of overt $\mathrm{PH}$ in some children, as especially evident in the setting of hypoplastic heart syndrome and single ventricle physiology.

CLASSIFICATION:The World Symposium on Pulmonary Hypertension (WSPH), which includes the Pediatric Task Force ${ }^{1}$ most recently updated the classification of PH-associated disorders in 2018 in Nice, France (Table 1). ${ }^{1}$ The role of the WSPH Classification is to improve understanding of underlying pathophysiology, natural history, therapeutic choices and clinical trial design. With regard to pediatric $\mathrm{PH}$, most pediatric PH patients are categorized in Class I (pulmonary arterial disease) and3 (hypoxia or lungassociated diseases). As reviewed in this supplement, growing awareness of the roles of PH in neonatal lung diseases and their sequelae, especially in association with bronchopulmonary dysplasia (BPD), congenital diaphragmatic hernia (CDH), persistent pulmonary hypertension of the newborn (PPHN), Down syndrome, and lethal developmental lung diseases, has led to greater appreciation of the unique nature of pediatric PH. ${ }^{1,4-8}$ Pharmacotherapy trials have almost exclusively been performed in patients with Class I PAH and almost entirely in adults, and the need for multicenter randomized clinical trials in children with these diverse forms of $\mathrm{PH}$ is further highlighted in this supplement. ${ }^{9}$ Based on the recognition of the differences in pediatric diseases and common co-morbidities that complicate $\mathrm{PH}$ outcomes, the pediatric committee of the international Pulmonary Vascular Research Institute (PVRI) proposed a more specific classification system for pediatric PVD during the meeting in Panama in 2011 (Table 2). ${ }^{10}$ This classification was derived independently from the WSPH Classification system to better reflect different etiologic categories of PH focused on infants, children and adolescents. However, the system is rather complex with overlapping categories, and Its adoption into clinical care and research has been mixed and not routinely applied.GENETICS PH is caused by both genetic and acquired factors, with key gene-environment interactions likely playing a major role in the pathobiology in many settings. Bone morphogenetic protein receptor II (BMPR2) is the most common genetic cause of PAH in adults, accounting for $~ 70 \%$ of familial cases and $10-20 \%$ of cases previously classified as idiopathic PAH. ${ }^{11}$ Ongoing discoveries continue to expand the list of genes associated with PAH. However, the genetic causes of pediatric PAH may differ from adults, and the causes of other types of PH are less well studied. In this supplement, Dr. Chung et al provide a critical overview regarding the importance of understanding the genetic basis of pediatric $\mathrm{PH}$, highlighting a different prevalence of specific genetic diseases in children in comparison with heritable etiologies in adult $\mathrm{PH} .{ }^{11}$ Importantly, the etiology of $\mathrm{PH}$ in infancy and early childhood often stems from developmental disorders of lung growth and development, which are associated with mutations in key genes involved in lung development, including FOXF1 (alveolar capillary dysplasia), TBX4, ACRVL1 and surfactant-related genes, as highlighted by Mallory et $\mathrm{al}^{6}$ in this supplement. 


\section{EPIDEMIOLOGY:}

It has been estimated that the prevalence of $\mathrm{PH}$ in pediatric populations in North America ranges from 20 to 64 cases per million with the majority of patients in WSPH class 1, some of whose PH improve with growth of the lung. ${ }^{1}$ Epidemiological studies of pediatric $\mathrm{PH}$ are derived from registry cohorts, and as a result, these data are affected by such factors as study design and the scope of clinical practice underlying patient selection for these registries. Geographic coverage, referral patterns, inclusion criteria and disease definitions may differ between registries, leading to potential selection bias. Overall, the estimated incidence of sustained $\mathrm{PH}$ in all categories was reported at 4-10 cases per million children per year with a prevalence of 20-40 cases per million. Importantly, more recent estimates have shown a striking proportion of infants in Group 3 or lung-related diseases, ${ }^{5}$ which often fall under the care of pediatric pulmonologists.

The prevalence of $\mathrm{PH}$ as a diagnosis on admission has significantly increased in the $21^{\text {st }}$ century as has the cost of hospitalization. ${ }^{15}$ Mortality is difficult to assess but in a recent ICU-based study, at least 144 pediatric $\mathrm{PH}$ patients were dying annually as recently as 2015 and this study excluded patients dying in neonatal ICU settings or prior to admission to the ICU. ${ }^{16}$ We project that 200- 500 pediatric patients with PH die per year in the USA and PH likely contributes to the cause of death in most. The understanding of the importance of PH in critical illness of the most severely ill patients in the ICU setting remains suboptimal, but issues in the management of severe right ventricular failure, the role of atrial septostomy or Potts shunt and possible lung transplantation for advanced disease is discussed below in this series. ${ }^{17-19}$ CLINICAL SPECTRUM :

It has been recognized for decades that the primary diseases, presentation and natural history of PH in children is quite different from adults, and is uniquely associated with the developmental biology of the lung and its circulation. ${ }^{3}$ In the era prior to the availability of targeted $\mathrm{PH}$ pharmacotherapy, the life expectancy of children newly diagnosed with $\mathrm{PH}$ was shorter than adults. ${ }^{20-22}$ Infants can often present with critical illness, unexplained hypoxemia, poor growth and unexplained respiratory distress. Older infants and children may have failure to thrive, recurrent seizures or exercise-induced syncopal episodes. As with adults, diagnosis can be unfortunately is delayed until the disease has advanced to a level of severity that includes right heart failure. The pediatric right ventricle appears to handle mechanical loads, summarized by pulmonary vascular resistance (PVR), before frank cor pulmonale is present. Similarly, exercise tolerance measured by six minute walk test is often maintained at a far later stage of the disease than in adults. ${ }^{22}$ The histopathology of PAH in children may differ from that in adults. Pathology tends to show a predominance of pulmonary arteriolar medial hypertrophy, less fibrosis and fewer intraluminal plexiform lesions in children, however, children can still develop aggressive structure remodeling as well. ${ }^{22}$

\section{UNAVOIDABLE USE OF "OFF LABEL" PHARMACOTHERAPY:}

There are now more 15 medications approved by the Food and Drug Administration (FDA) for the treatment of PAH, almost all of which are only formally approved for patients [?] 18 years of age. As with many disease processes, pediatric clinicians have employed many of these agents empirically in infants, children and adolescents, often with documented clinical benefit. Except for inhaled nitric oxide therapy (NO), most studies of current PH-targeted drugs are based on adult trials and only small case series in children. Problems related to the lack of sufficient data that address pediatric needs is exemplified by statements by the FDA on sildenafil use in children led to confusion and concern on the part of clinicians, insurers, and patient families. ${ }^{23}$

\section{CLINICAL CARE IN THE FACE OF RIGHT HEART FAILURE:}

The number of critically ill patients with significant PVD has increased in the past 20 years and pediatric critical care physicians have increasingly been involved in the care of these patients. ${ }^{17}$ These patients appear to be a particularly morbid subset of the overall ICU population. The use of inhaled nitric oxide has expanded even as its expense has not been definitively been addressed by the health care system. The use of systemic and inhaled prostacyclin medications has increased during this same era. The correct combination of targeted $\mathrm{PH}$ pharmacotherapeutic agents, vasopressors and other medications is currently 
tailored to individual patients by various institutional preferences and protocols. Randomized controlled studies are rare to non-existent related in part to the small numbers of patients in any given hospital and the heterogeneous nature of the pediatric $\mathrm{PH}$ population.

\section{PERSPECTIVES ON THE FUTURE}

As two senior clinicians in the field, we believe that we are in the midst an exciting era of clinical care and research, which includes novel opportunities to apply genomic, proteomic, metabolomic and imaging methodologies to enable rapid advances in the field of $\mathrm{PH}$. We believe that the manuscripts in this supplement should not only stimulate the interest of established pediatric pulmonologists but will hopefully attract our younger colleagues into this vibrant field. In addition, $\mathrm{PH}$ in neonates, infants and children is "a disease that knows no boundaries," which implies the clear need for developing interdisciplinary teams of pulmonologists, neonatologists, intensivists, cardiologists and others, to improve survival and enhance the quality of life for our children with $\mathrm{PH}$ and their families.

Bibliography:1. Rosenzweig EB, Abman SH, Adatia I, Beghetti M, Bonnet D, Haworth S, Ivy DD, Berger MRF. Paediatric pulmonary arterial hypertension: updates on definition, classification, diagnostics and management. Eur Resp J 2019; 53: doi: 10.1183/13993003.01916-2018.

2. Abman SH, Hansmann G, Archer S et al. American Heart Association and American Thoracic Society Joint Guidelines for Pediatric Pulmonary Hypertension. Circulation. 2015; 132:2037-99.

3. Abman SH, Raj U. Towards improving the care of children with pulmonary hypertension: rationale for developing a pediatric pulmonary hypertension network. Progress in Pediatr Cardiol, 2009; 27:3-6.

4. Whalen E, Ely E, Brown A. The Role of the Multidisciplinary Team in a Pediatric Pulmonary Hypertension Center. Pediatr Pulmonol. (this issue)

5. Varghese N, Tilman R, Keller R. Pulmonary Arterial Hypertension is an Important Co-Morbidity in Infant Lung Diseases: Bronchopulmonary Dysplasia and Congenital Diaphragmatic Hernia. Pediatr Pulmonol. (this issue)

6. Mallory GB, Spielberg D, Silva-Carmona M. Pulmonary Growth Abnormalities as Etiologies for Pediatric Pulmonary Hypertension. Pediatr Pulmonol. (this issue)

7. Bush D, Galambos C, ivy DD. Pulmonary Hypertension in Children with Down Syndrome. Pediatr Pulmonol. (this issue)

8. Mandell EW, Kinsella JP, Abman SH. Persistent Pulmonary Hypertension of the Newborn. Pediatr Pulmonol. (this issue)

9. Kam C, Fadel R. Opportunities and Challenges of Pharmacotherapy for Pulmonary Arterial Hypertension in Children. Pediatr Pulmonol. (this issue)

10. Cerro MJ, Abman S, Diaz G, Freudenthal AH, Freudenthal E, Harikrishan S, Haworth SG, Ivy D, Lopes AA, Ray JU, Sandoval J. Stenmark K, Adatia I. A consensus approach to the classification of pediatric pulmonary hypertensive vascular disease: report from the PVRI Pediatric Taskforce, Panama 2011. Pulm Circ 2011: 1: 286-298.

11. Welch C, Austin E, Chung W. Genes that Drive the Pathobiology of Pediatric Pulmonary Arterial Hypertension. Pediatr Pulmonol. (this issue)

12. del Cerro Marin MJ, Sabate Rotes A, Rodriguez Ogando A, et al. Assessing pulmonary hypertensive vascular disease in childhood. Data from the Spanish registry. Am J Respir Crit Care Med 2014; 190: $1421-1429$.

13. van Loon RL, Roofthooft MT, Hillege HL, et al. Pediatric pulmonary hypertension in the Netherlands. Epidemiology and characterization during the period 1991 to 2005. Circulation 2011; 124: 1755-1764. 
14. Li L, Jick S, Breitenstein S, et al. Pulmonary arterial hypertension in the USA: an epidemiological study in a large insured pediatric population. Pulm Circ 2017; 7: 126-136.

15. Awerbach JD, Mallory GB, Kim S, Cabrera AG. Hospital readmissions in children with pulmonary hypertension: a multi-institutional analysis. J Pediatr 2018, 195: 95-101.

16. Frank DB, Crystal MA, Morales DL, Gerald K, Hanna BD, Mallory GB Jr, Rossano JW Trends in pediatric pulmonary hypertension-related hospitalizations in the United States from 2000-2009. Pulm Circ $2015 ; 5: 339-48$.

17. Coleman R, Chartan C, Mourani P. Intensive Care Management of Right Ventricular Failure and Pulmonary Hypertension Crises. Pediatr Pulmonol. (this issue)

18. Grady R. Beyond transplant: Roles of Atrial Septostomy and Potts Shunt in Pediatric Pulmonary Hypertension. Pediatr Pulmonol. (this issue)

19. Melicoff E, Hayes D, Benden C. Lung transplantation as Intervention for Pediatric Pulmonary Hypertension. Pediatr Pulmonol. (this issue)

20. D'Alonzo GE, Barst RJ, Ayres SM, et al. Survival in patients with primary pulmonary hypertension: results from a national prospective registry. Ann Intern Med 1991; 115: 343-349.

21. Sandoval J, Bauerle O, Gomez A, Palomar A, Martinez Guerra ML, Furuya ME. Primary pulmonary hypertension in children: clinical characteristics and survival. J Am Coll Cardiol 1995; 25: 466-474.

22. Barst RJ, Ertel SI, Beghetti M, Ivy DD. Pulmonary arterial hypertension: a comparison between adults and children. Eur Respir J 2011; 37: 665-677.

23. Abman SH, Kinsella JP, Rosenzweig EB et al. Implications of the U.S. Food and Drug Administration warning against the use of sildenafil for the treatment of pediatric pulmonary hypertension. Am J Respir Crit Care Med 2013; 187: 572-575.

\section{Hosted file}

TABLE 1.pdf available at https://authorea.com/users/382570/articles/498475-editorial-themany-challenges-to-the-field-of-pediatric-pulmonology-posed-by-pediatric-pulmonaryhypertension-and-the-path-forward

\section{Hosted file}

Table 2.pdf available at https://authorea.com/users/382570/articles/498475-editorial-themany-challenges-to-the-field-of-pediatric-pulmonology-posed-by-pediatric-pulmonaryhypertension-and-the-path-forward 